\title{
General Entomology/Entomologia Geral Análise do nicho trófico e da influência das precipitações no forrageamento de Melipona eburnea Friese (Apidae: Meliponina) criadas no Acre, Brasil
}

\author{
Francisco Cildomar da Silva Correia ${ }^{\circledR}{ }^{\bowtie}$, Rui Carlos Peruquetti ${ }^{\&}$ Marcos Gonçalves Ferreira $^{2}$
}

1. Centro de Ciências Biológicas e da Natureza, Universidade Federal do Acre - UFAC. 2. Instituto Nacional de Pesquisas da Amazônia - INPA.

EntomoBrasilis 11 (1): 13-19 (2018)

\begin{abstract}
Resumo. O presente trabalho teve como objetivo identificar plantas utilizadas por Melipona eburnea Friese para coleta de pólen e verificar a influência das precipitações no forrageamento dessa espécie. Para isso, realizou-se duas coletas semanais, de outubro de 2015 a junho de 2016, em três colônias de M. eburnea. Abelhas operárias transportando cargas de pólen foram capturadas na entrada de suas colônias, utilizando-se rede entomológica. As cargas de pólen foram retiradas das corbículas das operárias com o auxílio de pinça e, posteriormente, levadas ao Laboratório para serem analisadas. Os grãos de pólen foram fotomicrografados e identificados através da comparação com o pólen das plantas em floração na área estudada. Os dados foram obtidos a partir do número de grãos de pólen nas amostras com a média de precipitação para cada mês. Identificouse 44 espécies botânicas, distribuídas em 18 famílias, as principais foram: Fabaceae, Myrtaceae, Euphorbiaceae e Arecaceae. Foi observado que a maior quantidade de pólen fornecido para M. eburnea ocorreu no período de menor volume pluviométrico. M. eburnea apresenta comportamento generalista na coleta de recursos polínicos, alterando seu nicho trófico, de acordo com a intensidade de floração. Plantas nativas representam a principal fonte de pólen para M. eburnea.
\end{abstract}

Palavras-chave: Abelha sem ferrão; Floração; Meliponicultura; Plantas meliponícolas; Recurso polínico.

\section{Analysis of the trophic niche and the influence of the precipitations on the foraging of Melipona eburnea Friese (Apidae: Meliponina) created in Acre, Brazil}

\begin{abstract}
The present work had as objective to identify plants used to collect pollen per Melipona eburnea Friese and to verify the influence of the precipitations in the foraging of this specie. For tath, two weekly collections were carried out, from October 2015 to June 2016, in three colonies of M. eburnea. Worker bees carrying pollen loads were captured at the entrance of their colonies, using an entomological net. The pollen loads were removed from the workers' corbulas with the aid of forceps and then taken to the laboratory for analysis. The pollen grains were photomicrographs and identified by comparison with the pollen of the flowering plants in the studied area. The data were obtained from the number of pollen grains in the samples with the average precipitation for each month. It was identified 44 pollen types distributed in 18 families, the main ones being: Fabaceae, Myrtaceae, Euphorbiaceae and Arecaceae. It was observed that the highest amount of pollen supplied to M. eburnea occurred in the period of lower rainfall volume. M. eburnea presents general behavior in the collection of pollinic resources. Native plants represent the main source of pollen for M. eburnea.
\end{abstract}

Keywords: Beekeeping plant; Flowering; Meliponiculture; Pollinic resource; Stingless bee.

$\mathbb{N}$ o Brasil são conhecidas atualmente 244 espécies de abelhas sem ferrão (PEDRo 2014), sendo que o maior número é encontrado na Amazônia legal (130 espécies) (Kerr et al. 2001; Silveira et al. 2002; Oliveira et al. 2013). Segundo Silva \& PAZ (2012), a criação desse grupo de abelhas é desenvolvida no país através da meliponicultura há muito tempo, especialmente nas regiões Norte e Nordeste. Para Silva et al. (2005), o meliponicultor, além de explorar o mel, pode alugar ou vender colônias, contribuindo, dessa forma, para a conservação de diversas espécies de meliponíneos e, consequentemente, ampliar seus lucros.
Na Amazônia brasileira são criadas, aproximadamente, 24 espécies e subespécies de abelhas sem ferrão (CoRTOPAssiLAURINo et al. 2006); Melipona eburnea Friese é uma delas. Essa espécie é endêmica de florestas tropicais e encontrada em, praticamente, toda a região amazônica (MAGALHÃes \& VENTURIERI 2010; PEDRo 2014), é utilizada em criação racional para extração de mel e outros subprodutos, por sua abundância na região, fácil adaptação em caixas e alta produtividade.

De modo geral, as abelhas sem ferrão estabelecem seus ninhos no interior de florestas, em cavidades de troncos de árvores vivas ou mortas (Michener 2000; RoubiK 2006). As atividades de voo
Edited by:

William Costa Rodrigues

\section{Article History:}

Received: 25.vii.2017

Accepted: 11.xi.2017
Corresponding author:

Francisco Cildomar da Silva Correia

○ cildomar12@hotmail.com

( ) No ORCID record
Funding agencies:

$\Delta$ Without funding declared 
são realizadas em busca de materiais para construção de suas colônias, para retirada do lixo produzido no interior das mesmas e, principalmente, para coleta de alimento, pólen e néctar (PICK \& BLOCHTEIN 2002). O pólen e o néctar são os recursos mais importantes para a maioria das abelhas, pois correspondem às principais fontes de proteínas e carboidratos para a manutenção de seus ninhos (Michener 1974; Roubik 1989). Por esse motivo, as abelhas estão entre os visitantes florais mais abundantes (RAMALHO et al. 1991).

As atividades de voo dos meliponíneos podem ser influenciadas pela oferta de recursos florais (pólen e néctar), condições internas das colônias (reserva de alimento e tamanho da população) e fatores abióticos (temperatura, umidade relativa, luminosidade, precipitação e velocidade do vento) (HILÁRIo et al. 2001; PICK \& BLOCHTEIN 2002), sendo as precipitações altamente danosas, pois afetam as atividades de coleta de alimento, obrigando as abelhas operárias a selecionarem os recursos mais próximos de suas colônias, alterando a dinâmica de forrageio (WINSTON 2003). No caso de as precipitações serem muito prolongadas, ainda interferem na produção de mel, visto que as abelhas operárias são forçadas a permanecerem mais tempo no ninho, aumentando o consumo do néctar armazenado (AlmeIDA et al. 2003). Outro aspecto é que maioria das plantas amazônicas floram no período seco do ano, facilitando, dessa forma, a ação de polinizadores e, como resultado, ocorre a conservação da diversidade (FRANKIE $e t$ al. 1974; WRIGHT \& CALDERON 1995).

Nesse sentido, o conhecimento das influências climáticas que interferem nas atividades de coleta de pólen, por parte das abelhas sem ferrão, faz-se importante para o estudo do comportamento desses indivíduos, visto que o conhecimento das atividades externas fornece indicação das condições gerais da colônia, podendo melhorar o desempenho da meliponicultura.

Assim, o objetivo deste trabalho foi identificar o pólen coletado por M.eburnea e, com isso, verificar a influência das precipitações nas atividades de forrageamento dessa espécie, contribuindo para o conhecimento de plantas nativas, exóticas e cultivadas, utilizadas por ela como fonte de alimento, gerando informações que podem incrementar práticas para o manejo dessa abelha e, consequentemente, a preservação da espécie.

\section{MATERIAL E MÉTODOS}

Local de estudo e dados climáticos. O estudo foi desenvolvido em Rio Branco - Acre, em um meliponário contendo 14 colônias de $M$. eburnea, mantidas em caixas padronizadas, modelo INPA, distribuídas em uma área de $600 \mathrm{~m}^{2}\left(09^{\circ} 56^{\prime} 6\right.$ "S; $67^{\circ} 53^{\prime} 6 ” \mathrm{~W}$ ) (Figura 1).

A propriedade possui 20 ha, sendo 16 formados por pastagens. Os quatro hectares restantes são formados por uma floresta secundária, apresentando espécies nativas em diferentes estágios de regeneração e um quintal com diversas plantas cultivadas e exóticas.

De acordo com a Classificação de KöPPEN (1931), o clima do Acre é do tipo equatorial, quente e úmido, com temperaturas médias anuais variando entre $24,5{ }^{\circ} \mathrm{C}$ e $32{ }^{\circ} \mathrm{C}$, ocorrendo duas estações distintas, uma seca e uma chuvosa. A estação chuvosa ocorre de novembro a abril, sendo caracterizada por chuvas constantes e abundantes e umidade relativa do ar atingindo 90\%. Já os índices pluviométricos variam de $1.600 \mathrm{~mm}$ a $2.750 \mathrm{~mm} / \mathrm{ano}$. A estação seca tem início em maio e se prolonga até outubro, ocorrendo, nesse período, o desaparecimento das chuvas, sendo comuns as friagens, que provocam quedas na temperatura de até $10{ }^{\circ} \mathrm{C}$.

Obtenção das amostras. Para amostragem dos dados foram realizadas duas coletas semanais, durante o período de outubro de 2015 a junho de 2016, em três colônias de M. eburnea. Abelhas operárias transportando cargas de pólen foram capturadas na entrada de suas colônias, utilizando-se rede entomológica. Os grãos de pólen foram retirados das corbículas das operárias com o auxílio de pinça e, posteriormente, levados ao Laboratório de Apoio à Vida Silvestre, da Universidade Federal do Acre, para serem analisados.

Identificação polínica e dados estatísticos. Os grãos de pólen foram fotomicrografados, utilizando-se microscópio óptico com câmera de três megapixels e acoplado a um computador equipado com programa de captura de imagens. Após, foram identificados, através da comparação com o pólen das plantas meliponícolas em floração na área estudada, e verificadas quais

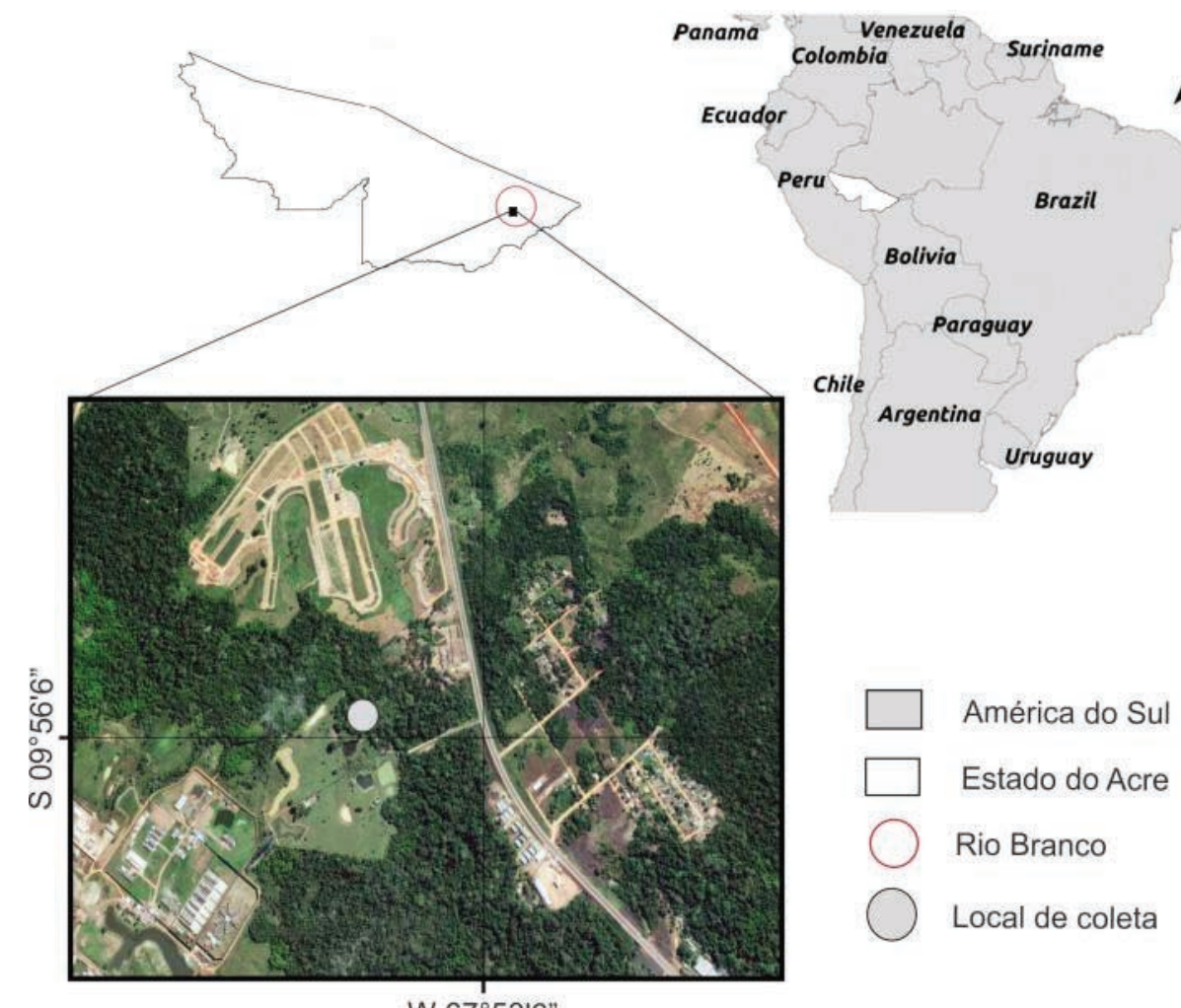

Figura 1. Local de coleta das amostras (Foto: Google Earth). 
espécies forneceram esse recurso para as abelhas dentro de cada mês, através da contagem dos grãos presentes nas amostras, utilizando a metodologia descrita por (Colinvaux et al. 1999), sendo os resultados expressos aqui em porcentagem.

Os dados de correlação foram obtidos a partir do número de grãos de pólen que ocorreram em cada amostra mensal, com a média de precipitação observada para cada mês. Após identificados, os grãos de pólen foram classificados como pertencentes à plantas nativas, cultivadas e exóticas. A correlação linear entre tais grupos botânicos e precipitação foi verificada através do programa estatístico Past (Paleontological statistics) versão 2.17c (HAMmer et al. 2001), com nível de significância de 95\%.

Os dados meteorológicos foram obtidos no site do Instituto Nacional de Meteorologia (INMET - http://goo.gl/GIe6Y8; estação meteorológica convencional 82915).

\section{RESULTADOS}

As 44 espécies botânicas identificadas foram distribuídas em 18 famílias, sendo as principais: Fabaceae, com 16 espécies, seguida por Myrtaceae e Euphorbiaceae com cinco cada e Arecaceae com três (Tabela 1).

Tabela 1. Registro da frequência de plantas nativas, cultivas e exóticas, com base nos grãos de pólen coletados por Melipona eburnea, de outubro de 2015 a junho de 2016. Plantas meliponícolas (PM), nativas (n), cultivadas (c), exóticas (e) e em nível de família (gênero e espécie indefinidos) (i).

\begin{tabular}{|c|c|c|c|c|c|c|c|c|c|}
\hline \multirow{2}{*}{ FAMÍLIA/planta } & \multicolumn{3}{|c|}{2015} & \multicolumn{6}{|c|}{2016} \\
\hline & Out & Nov & Dez & Jan & Fev & Mar & Abr & Mai & Jun \\
\hline
\end{tabular}

\section{AMARANTHACEAE}

Chenopodium ambrosioides $\mathrm{L}$.

$\mathrm{X}$

ARECACEAE

Cocos nucifera L.

Euterpe oleracea Mart.

Oenocarpus bacaba Mart.

BIXACEAE

Bixa orellana $\mathrm{L}$.

BORAGINACEAE

Cordia nodosa Lam.

EUPHORBIACEAE

Alchornea sp.

Aparisthmium cordatum (A. Juss.) Baill.

Croton sp.

Euphorbiaceae

Manihot esculenta Crantz

FABACEAE

Acacia polyphylla DC.

Acacia sp.

Caesalpinia L.

Cassia sp.

$\mathrm{X}$

$\mathrm{X}$

Crotalaria retusa L.

Enterolobium schomburgkii Benth.

Inga edulis Mart.

Inga emarginata Humb. \& Bonpl. Ex Willd.

Mimosa adenophylla Taub.

Mimosa caesalpiniifolia Benth.

Mimosa pigra L.

Mimosa pudica L.

Mimosa setosa Benth.

Pithecellobium Mart.

Schizolobium parahyba var. amazonicum (Huber ex Ducke) Barneby

Swartzia sp.

MALPIGHIACEAE

Malpighia emarginata DC.

$\mathrm{X} \quad \mathrm{X}$

MARANTACEAE

Calathea sp.

MELASTOMATACEAE

Miconia sp.

MYRTACEAE

Eugenia jambolana Lam.

Eugenia sp.

Myrtaceae

Psidium guajava L.

Syzygium malaccense (L.) Merr. \& LM Perry

MORACEAE

Ficus sp.

$x$

X

$\mathrm{X}$

Musa sapientum L.

POACEAE

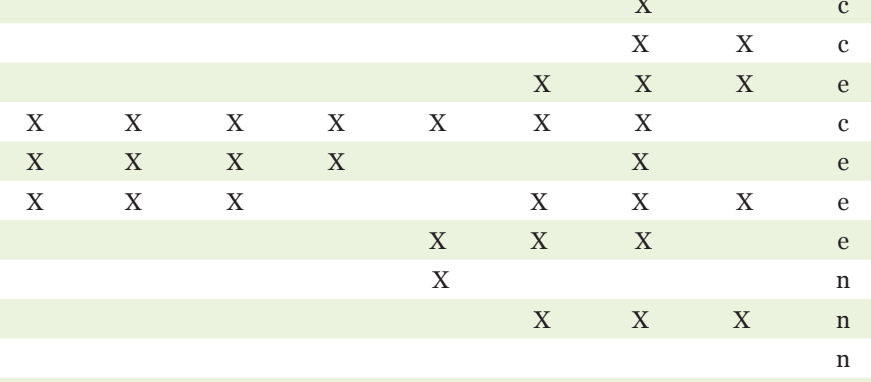


Tabela 1. Continuação...

\begin{tabular}{|c|c|c|c|c|c|c|c|c|c|c|}
\hline \multirow{2}{*}{ FAMÍLIA/planta } & \multicolumn{3}{|c|}{2015} & \multicolumn{6}{|c|}{2016} & \multirow[t]{2}{*}{$\mathbf{P M}$} \\
\hline & Out & Nov & Dez & Jan & Fev & Mar & Abr & Mai & Jun & \\
\hline Zea mays $\mathrm{L}$. & & $\mathrm{X}$ & $\mathrm{X}$ & & $\mathrm{X}$ & & & & & \\
\hline RUBIACEAE & & & & & $\mathrm{X}$ & & & & & $\mathrm{i}$ \\
\hline
\end{tabular}

RUTACEAE

Citrus x limon (L.) Osbeck

$\mathrm{X}$

SAPINDACEAE

Allophylus L.

Cupania sp

$\mathrm{X}$

SMILACACEAE

$\mathrm{X}$

$\begin{array}{llll} & & n \\ X & X & n\end{array}$

Smilax sp.

$\mathrm{X}$

SOLANACEAE

Solanum sp.

De acordo com as observações realizadas neste estudo, podemos destacar os períodos de floração e os meses com maior riqueza de plantas poliníferas para M. eburnea, sendo o mais abundante, maio de 2016 (17 espécies); seguido por abril de 2016 (15 espécies), novembro e dezembro de 2015 (14 espécies) e fevereiro e junho de 2016 (13 espécies). Conforme o grau de importância, podemos ressaltar que, das 44 plantas coletadas por M. ebúrnea, 19 espécies $(43,2 \%)$ foram oriundas da flora nativa, $16(36,4 \%)$ de plantas cultivadas e seis $(13,6 \%)$ de plantas exóticas. As demais plantas só foram classificadas em nível de família (Euphorbiacea, Myrtaceae e Rubiaceae) e totalizaram 6,8\% (Tabela 1).

Quanto à importância das plantas e ao período de floração, podemos ressaltar que as menos sazonais foram: Solanum sp. (Solanaceae), ocorrendo em todo o período de coleta; Mimosa caesalpiniifolia Benth. (Fabaceae), ocorrendo de novembro de 2015 a maio de 2016; Miconia sp. (Melastomataceae), de janeiro a junho de 2016; Crotalaria retusa L. (Fabaceae), de fevereiro a junho de 2016; Myrtaceae e Eugenia jambolana Lam. (Myrtaceae), de outubro de 2015 a fevereiro de 2016 e Manihot esculenta Crantz (Euphorbiaceae), de março a junho de 2016, como pode ser confirmado na Tabela 1.

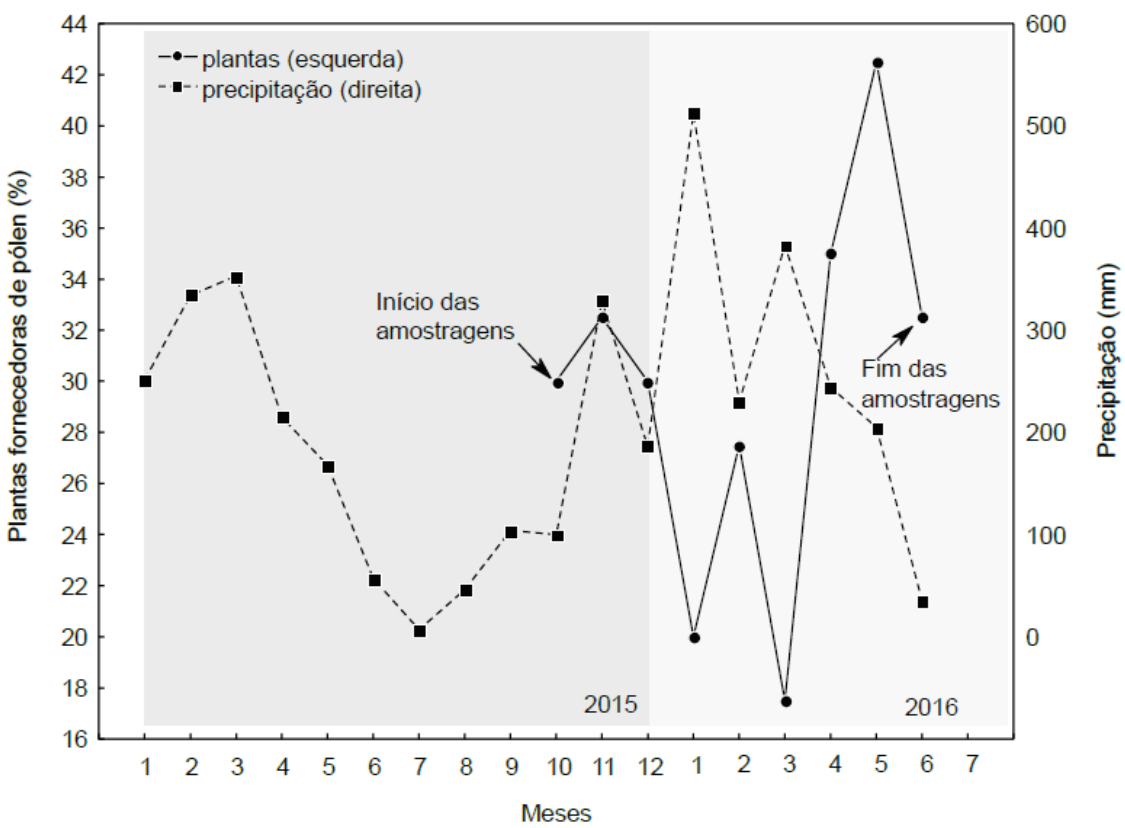

Figura 2. Dados de precipitações e plantas utilizadas por Melipona eburnea para coleta de pólen, de outubro de 2015 a junho de 2016.

Tabela 2. Variação da coleta de pólen por Melipona eburnea entre plantas nativas, cultivadas e exóticas, de outubro de 2015 a junho de 2016.

\begin{tabular}{|c|c|c|c|c|c|c|c|c|c|c|}
\hline Espécies & Out & Nov & Dez & Jan & Fev & Mar & Abr & Mai & Jun & Total \% \\
\hline Nativas & 6,0 & 5,1 & 6,0 & 3,5 & 5,1 & 3,5 & 5,1 & 5,1 & 5,1 & 42,8 \\
\hline Cultivadas & 4,3 & 4,3 & 4,3 & 1,7 & 3,4 & 1,7 & 3,4 & 3,0 & 3,5 & 31,3 \\
\hline Exóticas & & 1,7 & 1,7 & 1,7 & 1,7 & 1,7 & 3,4 & 5,1 & 2,6 & 19,6 \\
\hline Indeterminadas & 0,9 & 0,9 & 0,9 & 0,9 & 1,8 & & 0,9 & & & 6,3 \\
\hline
\end{tabular}




\section{DISCUSSÃO}

Resultados semelhantes aos observados neste estudo foram registrados em uma análise realizada na Colômbia por OBREGON \& NATES-PARRA (2014), com a mesma espécie de abelha. Esses autores identificaram 92 espécies botânicas, pertencentes a 38 famílias, desse total $68 \%$ vinham de plantas nativas. Quanto aos tipos polínicos identificados nas análises, os mais frequentes foram: Asteraceae (20,4\%), Myrtaceae (20,0\%), Euphorbiaceae $(16,7 \%)$ e Solanaceae (13,3\%).

FreitAs et al. (2010) observaram que as famílias Fabaceae, Euphorbiaceae, Melastomataceae, Myrtacea e Solanaceae estão entre as famílias botânicas com maior frequência nos levantamentos de flora meliponícola, realizados no Brasil e na Venezuela, corroborando com os resultados encontrados nesta investigação.

Com relação aos índices pluviométricos, Belavadi \& GaneshaiAH (2013) salientam que, em estações com elevado número de precipitações (muitos dias chovendo), há diminuição do número de plantas floridas, principalmente se as chuvas ocorrerem no período matutino, uma vez que este intervalo corresponde ao de maior atividade de coleta de recursos polínicos por M. eburnea (Nates-Parra \& Rodrígues 2011). Pierrot \& Schlindwein (2003) destaca que a quantidade de pólen disponível nas anteras das flores diminui drasticamente durante a estação chuvosa, devido à lavagem realizada pela chuva. O tempo de forrageamento e a atividade de voo também são prejudicados pelas precipitações (Almeida et al. 2003).

Nesse sentido, a Figura 3 mostra a interferência dos índices pluviométricos na quantidade de pólen ofertado a $M$. eburnea por espécies nativas, visto que $\left(\mathrm{r}^{2}=0,769 \mathrm{p}=0,015\right)$, evidenciando que quanto menor o índice de precipitações, maior o número de tipos polínicos oriundos dessas espécies de plantas.

Durante o mês de maio de 2016 foi observado que as abelhas coletaram pólen de uma maior variedade de plantas (Figura 1), destacando-se as espécies nativas, visto que, em florestas tropicais, a floração da maioria das espécies acontece nos meses com menor ocorrência de precipitações, devido ao favorecimento à ação de polinizadores (Frankie et al. 1974); (Wright \& CALDERoN
1995). Em contrapartida, cabe salientar que em estudos polínicos realizados em área de várzea, na Amazônia central, com abelhas do gênero Melipona (FerReira \& ABSY 2015) foi demonstrado que durante o período chuvoso ocorre intensificação de floração e, consequentemente, maior riqueza de grãos de pólens coletados por esse grupo de abelhas. Entretanto, essa intensa floração durante o período chuvoso pode, em determinadas áreas, como mostrado nesse estudo, ser diminuída ou composta por plantas que não são meliponícolas.

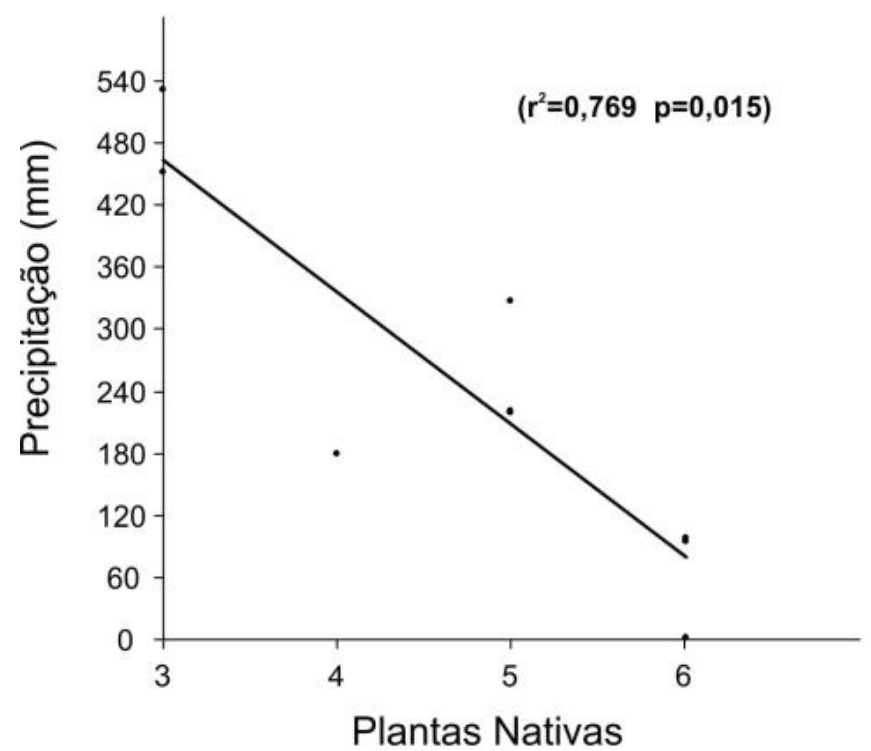

Figura 3. Correlação entre o índice de precipitação e plantas nativas utilizadas para coleta de pólen por Melipona eburnea, de outubro de 2015 a junho de 2016.

Para as plantas cultivadas, o resultado se apresentou semelhante ao das plantas nativas $\left(\mathrm{r}^{2}=0,427 \mathrm{p}=0,056\right)$, mostrando que baixos índices pluviométricos favorecem a oferta de pólen dessas plantas (Figura 4).

A exceção foi a correlação entre precipitação e plantas exóticas utilizadas por M. eburnea, pois não apresentaram resultado

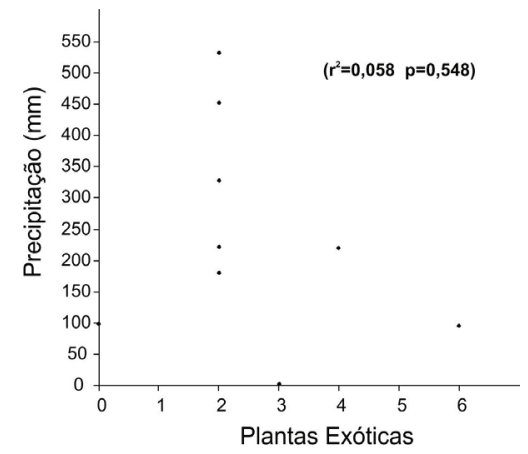

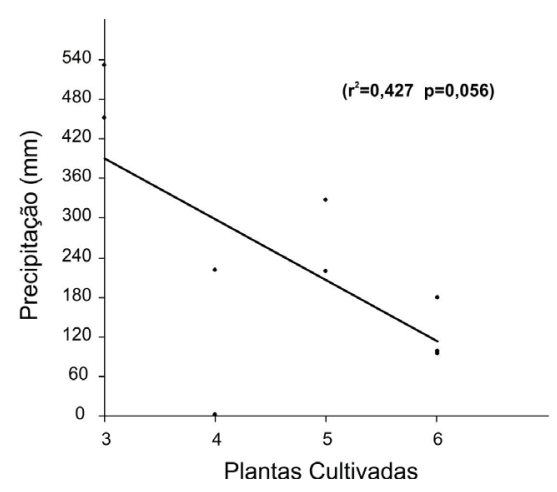

Figura 4. Correlação entre índice pluviométrico e plantas cultivadas, utilizadas para coleta de pólen por Melipona eburnea, de outubro de 2015 a junho de 2016. 
significativo, tendo em vista que $\left(\mathrm{r}^{2}=0,058 \mathrm{p}=0,548\right)$ (Figura $5)$.

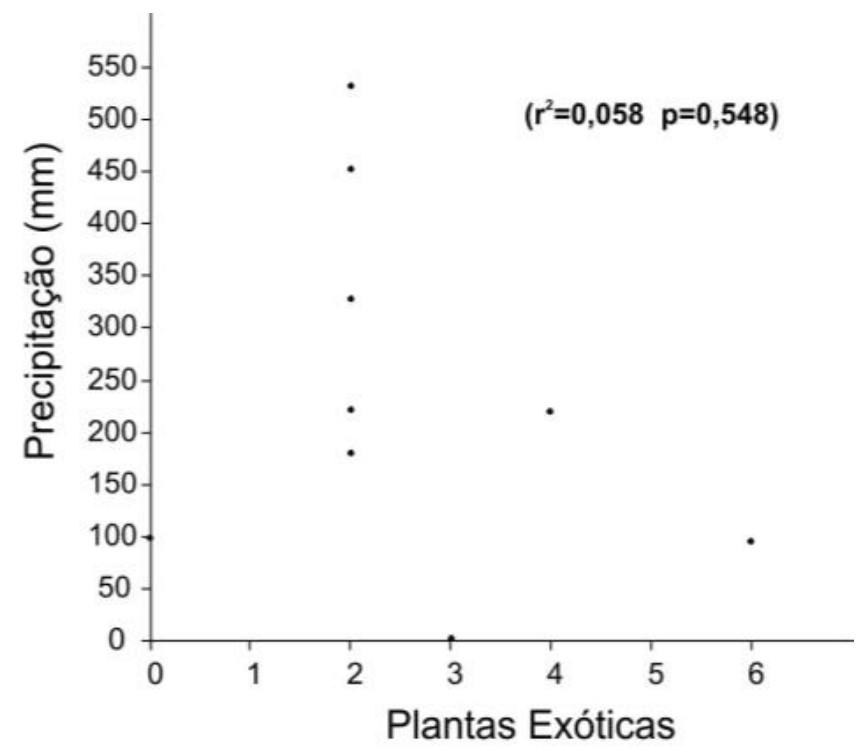

Figura 5. Correlação entre índice pluviométrico e plantas exóticas, utilizadas para coleta de pólen por Melipona eburnea, de outubro de 2015 a junho de 2016.

Quanto às plantas classificadas em nível de família, não se verificou a correlação entre elas e os índices pluviométricos, devido a não identificação de gênero e espécie.

Segundo Bawa et al. (1989); Tuell \& IsaAcs (2010); Belavadi \& Geneshaiah (2013), na época chuvosa, o pólen disponível pode não ser acessado pelas abelhas, seja pela alta umidade do ar ou pela precipitação, que dificulta os voos de forrageamento. HILÁRIO et al. (2007), observando o impacto da ação pluviométrica sobre a atividade de voo de Plebeia remota Holmberg, verificaram queda na atividade de voo dessa abelha, antes e durante a chuva. Contudo, é possível que chuvas no período matutino sejam particularmente danosas às atividades de coleta de pólen pelas operárias de $M$. eburnea, pois esta espécie de abelha costuma coletar pólen nas primeiras horas do dia.

Em um estudo realizado por TuELL \& IsAACs (2010), observando as atividades de forrageio de abelhas produtoras de mel, entre maio de 2007 e maio e junho de 2008, verificou-se que a coleta de pólen foi prejudicada mediante a exposição às péssimas condições meteorológicas. Em outra análise, realizada por NAScIMENTO \& NAscimento (2012), com três colônias de Melipona asilvai Moure entre duas estações do ano, chuvosa e seca, percebeu-se diferenças visíveis nas atividades de forrageio desta espécie de abelha, durante a estação chuvosa houve redução acentuada do número de operárias, saindo de seus ninhos para coleta de recursos. Para Almeida et al. (2003), a chuva é de grande importância para a prefloração, pois assegura condições necessárias a uma floração eficiente. No entanto, em plena florada, são prejudiciais à produção de mel, já que as abelhas não saem da colônia para suas atividades de forrageio e ainda consomem parte do mel já armazenado.

Belavadi \& Ganeshaiah (2013) afirmam que, além da chuva, outros fatores ambientais como temperatura, umidade, velocidade do vento, intensidade da luz e nebulosidade podem afetar o comportamento de floração das plantas e atividade de forrageamento do polinizador.

Neste sentido, observações realizadas por Michenter (1974); SOMMEIJER et al. (1983); KLEINERT-GIOVANNINI \& IMPERATrIZFonseca (1986); Roubik (1989); Hilário et al. (2001); Pick \& BLOCHTEIN (2002); KAJOBE \& ECHAZARRETA (2005) verificaram que precipitações pluviométricas causam diminuição ou interrupção de atividades externas à colônia.
Diante disso, foi possível constatar que, em meses com alto índice pluviométrico, houve menor oferta de recursos polínicos para $M$. eburnea. Tal fato pode estar relacionado com a dificuldade de forrageamento da abelha durante esse período, já que, em condições extremas de precipitação, sua capacidade de voo pode ser prejudicada, diminuindo a entrada de pólen na colônia e, consequentemente, afetando o desenvolvimento do meliponário.

Foi verificado que $M$. eburnea apresenta comportamento generalista no uso das fontes de pólen, alterando seu nicho trófico de acordo com a oferta desse recurso.

Plantas nativas representam a principal fonte de recursos polínicos para M. eburnea. Também foi identificado qu em meses com elevado índice de precipitações (muitos dias chovendo), ocorre a diminuição da oferta de pólen para $M$. eburnea.

\section{REFERÊNCIAS}

Almeida, D.L., Marchini, C., Sodré, G.S., D’Ávila, M. \& C.M.F. Arruda, 2003. Plantas visitadas por abelhas e polinização. São Paulo, Esalq, 40 p.

Bawa, K.S., Hegde, S.G. Ganeshaiah, K.N. \& R.U. Shaanker, 1989. Embryo and seed abortion in plants. Nature, 342: 625-626. DOI: https://doi.org/10.1038/342625ao.

Belavadi, V.V. \& K.N. Ganeshaiah, 2013. Insect pollination manual. Department of Agricultural Entomology University of Agricultural Sciences. Bangalore, New Delhi, 44 p.

Colinvaux, P.A., Oliveira, P.E. \& J.E. Moreno, 1999. Amazon Pollen Manual and Atlas. New York, Harwood Academic Press, 344 p.

Cortopassi-Laurino, M., Imperatriz-Fonseca, V.L., Roubik, D.W., Dollin, A., Heard, T., Aguilar, I.B., Venturiere, G.C., Eardley, C. \& P. Nogueira-Neto, 2006. Global Meliponiculture: challenges and opportunities. Apidologie, 37: 275-292. DOI: https://doi.org/10.1051/apido:2006027.

Ferreira, M.G. \& M.L. Absy, 2015. Pollen niche and trophic interactions between colonies of Melipona (Michmelia) seminigra merrillae and Melipona (Melikerria) interrupta (Apidae: Meliponini) reared in floodplains in the Central Amazon. Arthropod Plant Interactions, 9: 263-279. DOI: https://doi.org/10.1007/s11829-015-9365-0.

Frankie, G.W., Baker, H.G. \& P.A. Opler, 1974. Comparative phenological studies of trees in tropical wet and dry forests in the lowlands of Costa Rica. Journal of Ecology, 62: 881-913. DOI: https://doi.org/10.2307/2258961.

Freitas, A.S. Barth, O.M. \& C.F.P. Luz, 2010. Análise polínica comparativa e origem botânica de amostras de mel de Meliponinae (Hymenoptera, Apidae) do Brasil e da Venezuela. Mensagem Doce, 106: 2-9. Disponível em: <https://www. researchgate.net/publication/235418391 Analise polinica comparativa e origem botanica de amostras de mel de Meliponinae Hymenoptera Apidae do Brasil e da Venezuela>.

Hammer, Ø., Harper, D.A.T. \& P.D. Ryan, 2001. PAST: Paleontological Statistics software package for education and data analysis. Palaeontologia Electronnica, 4: 1-9. Disponível em: <http://palaeo-electronica.org/2001 1/past/past.pdf >.

Hilário, S.D.; Ribeiro, M.F. \& V.L. Imperatriz-Fonseca, 2007. Impacto da precipitação pluviométrica sobre a atividade de voo de Plebeia remota (Holmberg, 1903) (Apidae, Meliponini). Biota Neotropica, 7: 135-143. DOI: https://doi.org/10.1590/ s1676-06032007000300016.

Hilário, S.D., Imperatriz-Fonseca, V.L. \& A. KleinertGiovannini, 2001. Responses to climatic factors by foragers of Plebeia pugnax Moure (IN LITT) (Apidae, Meliponinae). Revista Brasileira de Biologia, 61: 191-196. DOI: https://doi.org/10.1590/s0034-71082001000200003.

Kajobe, R. \& C.M. Echazarreta, 2005. Temporal resource partitioning and climatological influences on colony flight and foraging of stingless bees (Apidae, Meliponini) in Ugandan 
tropical forests. African Journal Ecoogical, 43: 267-275. DOI: https://doi.org/10.1111/j.1365-2028.2005.00586.x.

Kerr, W.E., Carvalho, G.A., Silva, A.C. \& M.G.P. Assis, 2001. Aspectos pouco mencionados da biodiversidade amazônica. Parcerias Estratégicas, 6: 20-41. Disponível em: $<$ http://seer.cgee.org.br/index.php/parcerias estrategicas/ article/view/183>.

Kleinert-Giovannini, A. \& V.L. Imperatriz-Fonseca, 1986. Flight activity andresponses to climatic conditions of two subspecies of Melipona marginata Lepeletier (Apidae, Meliponinae). Journal of Apicultural Research, 25: 3-8. DOI: https://doi.org/10.1080/00218839.1986.11100685.

Köppen, W., 1931. Grundriss der Klimakunde. Berlin, Walter de Gruyter, $390 \mathrm{p}$.

Magalhães, T.L. \& G.C. Venturieri, 2010. Aspectos econômicos da criação de abelhas indígenas sem ferrão (Apidae: Meliponini) no nordeste paraense. Belém, Embrapa Amazônia Oriental, $364 \mathrm{p}$.

Michener, C.D., 2000. The bees of the world. Baltimore, The Johns Hopkins University Press, 913 p.

Michener, C.D., 1974. The Social Behavior of the Bees: A Comparative Study. Massachussets, The Belknap Press of Harvard University Press, 404 p.

Nascimento, D.L. \& F.S. Nascimento, 2012. Extreme Effects of Season on the Foraging Activities and Colony Productivity of a Stingless Bee (Melipona asilvai Moure, 1971) in Northeast Brazil. Hindawi Publishing Corporation Psyche, 2012: 1-6. DOI: https://doi.org/10.1155/2012/267361.

Nates-Parra, G. \& C. Rodriguez, 2011. Forrajeo em colonias de Melipona eburnea (Hymenoptera: Apidae) em el piedemonte llanero (Meta, Colômbia). Revista Colombiana de Entomologia, 37: 121-127. Disponível em: <http://www.scielo.org.co/pdf/ rcen/v37n1/v37n1a22.pdf $>$.

Obregon, D. \& G. Nates-Parra, 2014. Floral Preference of Melipona eburnea Friese (Hymenoptera: Apidae) in a Colombian Andean Region. Neotropical Entomology, 43: 536o. DOI: https://doi.org/10.1007/s13744-013-0172-y.

Oliveira, F.F., Richers, B.T.T., Silva, J.R., Farias, R.C. \& T.A.L. Matos, 2013. Guia Ilustrado das abelhas sem ferrão das Reservas Amanã e Mamirauá, Brasil (Hymenoptera, Apidae, Meliponini). Tefé, Instituto de Desenvolvimento Sustentável Mamirauá, $267 \mathrm{p}$.

Pedro, S.R.M., 2014. The Stingless Bee Fauna. In Brazil (Hymenoptera: Apidae). Sociobiology, 61: 348-354. DOI: https://doi.org/10.13102/sociobiology.v61i4.348-354.

Pierrot, L.M. \& C. Schlindwein, 2003. Variation in daily flight activity and foraging patterns in colonies of uruçu - Melipona scutellaris Latreille (Apidae, Meliponini). Revista Brasileira de Zoologia, 20: 565-571. DOI: https://doi.org/10.159o/ s0101-81752003000400001.

Pick, R.A. \& B. Blochtein, 2002. Atividades de coleta e origem floral do pólen armazenado em colônias de Plebeia saiqui (Holmberg) (Hymenoptera, Apidae, Meliponinae) no sul do Brasil. Revista brasileira Zoologia, 19: 289-300. DOI: https://doi.org/10.1590/s0101-81752002000100025.

Ramalho, M., Imperatriz-Fonseca. V.L. \& A. Kleinert-Giovannini. 1991. Ecologia nutricional de abelhas sociais, p. 225-252. In: Panizzi, A.R. \& J.R.P. Parra, (Orgs.), Ecologia nutricional de insetos e suas implicações no manejo de pragas. São Paulo, Manole, $359 \mathrm{p}$.

Roubik, D.W. 2006. Stingless bee nesting biology. Apidologie, 37: 124-143. DOI: https://doi.org/10.1051/apido:2006026.

Roubik, D.W., 1989. Ecology and natural history of tropical bees. New York, Cambridge University Press, 514 p.

Silva, W.P. \& J.R.L. Paz, 2012. Abelhas sem ferrão: muito mais do que uma importância econômica. Natureza on line, 10: 146-152. Disponível em: <http://www.naturezaonline.com. $\mathrm{br} /$ natureza/conteudo/pdf/og Silva Paz 146152.pdf $>$.

Silveira, F.A., Melo G. A. R. \& E.A.B. Almeida, 2002. Abelhas brasileiras - sistemática e identificação. Belo Horizonte, Composição e Arte, 253 p.

Silva, E.M.S., Freitas, B.M., Silva, L.A., Cruz, D. O. \& I.G.A. Bomfim, 2005. Biologia floral do pimentão (Capsicum annuum) e a utilização da abelha jandaíra (Melípona subnitida Ducke) como polinizador em cultivo protegido. Revista Ciência Agronômica, 36: 386-390. Disponível em: $<$ http://ccarevista.ufc.br/seer/index.php/ccarevista/article/ view $/ 256>$.

Sommeijer, M.J., de Rooy, G.A. Punt, W. \& L.L.M. Bruijn, 1983. A comparative study of foraging behaviour and pollen resources of various stingless bees (Hym., Meliponinae) and honey bees (Hym., Apinae) in Trinidad, West-Indies. Apidologie, 14: 205-224. DOI: https://doi.org/10.1051/apido:19830306.

Tuell, J.K. \& R. Isaacs, 2010. Weather During Bloom Affects Pollination and Yield of Highbush Blueberry. Entomological Society of America, 103: 205-224. DOI: https://doi.org/10.1603/ec09387.

Winston, M. L., 2003. A biologia da abelha. Porto Alegre, Magister, $427 \mathrm{p}$.

Wright, S.J. \& O. Calderon, 1995. Phylogenetic patterns among tropical flowering phenologies. Journal of Ecology, 83: 937948. DOI: https://doi.org/10.2307/2261176.

\section{Suggestion citation:}

Correia, F.C.S., R.C. Peruquetti \& M.G. Ferreira, 2018. Análise do nicho trófico e da influência das precipitações no forrageamento de Melipona eburnea Friese (Apidae: Meliponina) criadas no Acre, Brasil. EntomoBrasilis, 11 (1): 13-19.

Available on: doi:10.12741/ebrasilis.v11i1.711
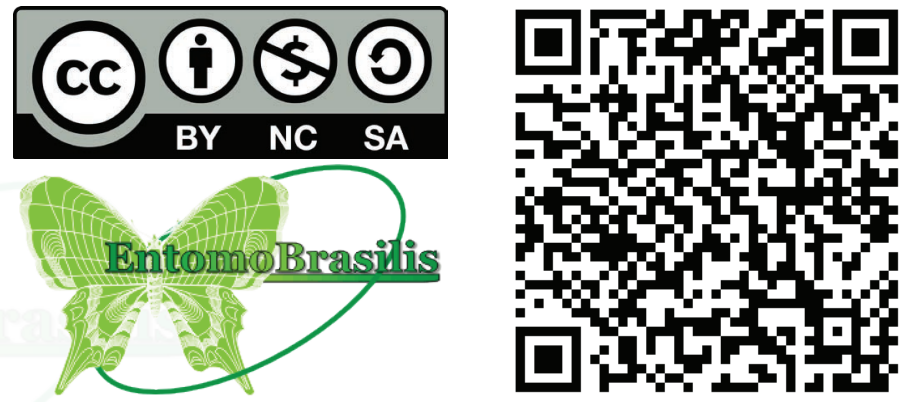